\title{
Enfermedad pilonidal sacro-coccígea: factores de riesgo y tratamiento quirúrgico*
}

\author{
Dr. GUILLERMO BANNURA C. \\ 1 Servicio y Departamento de Cirugía, Hospital Clínico San Borja Arriarán. Facultad de Medicina, \\ Universidad de Chile. \\ Santiago, Chile.
}

\section{Sacrococcygeal pilonidal disease. Risks factors and surgical options}

La enfermedad pilonidal sacrococcígea (EPSC) es una condición adquirida frecuente que afecta principalmente a adultos jóvenes del género masculino ${ }^{1}$. Tanto la causa como el mejor tratamiento para esta condición son temas de debate. En esta breve revisión discutiremos algunos factores considerados como de riesgo para adquirir la enfermedad y nuestra elección entre las alternativas quirúrgicas actuales.

\section{Factores de riesgo de la EPSC}

Los factores etiopatogénicos de la EPSC son aún motivo de controversia. La real incidencia de la EPSC es desconocida, pero un estudio efectuado en Noruega estima esta cifra en 25 x 100.000 habitantes ${ }^{2}$. Se considera rara en Asia y en la población negra ${ }^{1}$. Aunque afecta predominante a hombres jóvenes, según este estudio la relación mujer/hombre es 4:1 en el grupo etario preadolescente ${ }^{2}$. Por otra parte, en Chile hasta la década del 80 se destacaba una clara predominancia de las mujeres en una proporción de $3: 1^{3-7}$, situación que al parecer ha cambiado y en nuestra serie prospectiva de 145 pacientes intervenidos en los últimos 9 años la relación hombre/ mujer es $1: 1$.

Actualmente, se acepta que la EPSC es una con- dición adquirida que no corresponde a un quiste ${ }^{8}$. En realidad se trata de un absceso crónico causado por bacterias que ingresan al tejido graso subcutáneo a través de los folículos pilosos ensanchados (poros) de la piel a nivel de la línea media de la hendidura entre los glúteos ${ }^{9}$. Estos poros se producen por el efecto de tracción hacia abajo de los glúteos que ejercen una formidable fuerza sobre la piel que está fija al hueso coccígeo, especialmente al ponerse de pie y al sentarse. Secundariamente, se produce la penetración del invasor ${ }^{10}$, es decir, el pelo, que provoca una infección persistente de bajo grado, el absceso crónico, secundariamente la formación de tejido granulatorio y, en el extremo del abanico, la reacción gigantocelular a cuerpo extraño. Se ha demostrado claramente que el pelo no ingresa por la raíz, confirmando que se trata de pelos sueltos de la región lumbar y de la espalda que son eliminados en forma natural ${ }^{11}$. En algunos pacientes un manojo de pelos puede ser succionado hacia la línea media por la presión negativa o el vacío que se crea entre los glúteos. Como el surco interglúteo es profundo y húmedo, particularmente en personas con sobrepeso, la obesidad ha sido identificada como un factor de riesgo de desarrollar la EPSC ${ }^{2,12,13}$. Del mismo modo, el hirsutismo se ha considerado como un factor predisponente de esta condición ${ }^{1,13,14}$, habitualmente denominada 'quiste pilonidal' (en latín, nido de pe-

* Recibido el 28 de marzo de 2011 y aceptado para publicación el 11 de mayo de 2011.

Correspondencia: Dr. Guillermo Bannura C.

Las Limas 1622, Santiago, Chile.

gbannura@vtr.ne 
los). Para apoyar esta teoría, se ha señalado la mayor incidencia de esta enfermedad en varones jóvenes desaseados de pelo grueso, destacando la gran cantidad de soldados intervenidos en la segunda Guerra Mundial (jeep disease) ${ }^{15}$. Sin embargo, la EPSC con frecuencia se ve en pacientes de peso normal, en personas lampiñas y, como ya se comentó, en las series nacionales históricas la incidencia incluso fue mayor en mujeres, las que habitualmente son menos hirsutas. Por estos motivos, quisimos evaluar la relevancia de la obesidad y del hirsutismo como factores etiopatogénicos de la EPSC en nuestro medio, así como su eventual relevancia en la morbilidad y la recidiva de la enfermedad.

En el mes de mayo de 2001 iniciamos un protocolo prospectivo mediante la confección de una base de datos que registró los antecedentes demográficos de los pacientes portadores de una EPSC, las intervenciones previas, la forma de presentación clínica, la técnica quirúrgica empleada y la morbilidad. Se utilizó la técnica de resección asimétrica de Karydakis $^{10,11,16}$ en los casos habituales, reservando la plastía de Limberg para los casos recidivados y aquellos pacientes con múltiples orificios fistulosos y compromiso glúteo. Se excluyen los casos agudos tratados con simple drenaje por un absceso pilonidal. No se utilizó preparación mecánica del colon. Durante la inducción anestésica se indicó una dosis única de cloxacilina 1 gramo iv con fines de profilaxis.

Para evaluar el grado de obesidad de los pacientes se calculó el índice de masa corporal (IMC) al ingreso dividiendo el peso en kilos por la estatura en metros al cuadrado. El IMC se analizó como variable continua y como variable categórica, definiendo las categorías normal (IMC $=18,5-24,9$ ), sobrepeso (IMC $=25-29,9)$ y obeso (IMC mayor de 30). Para los efectos de este estudio, se define hirsutismo como una variable categórica: presencia de pelo grueso y oscuro en la región lumbar y en la espalda (sí/no). El grupo control está compuesto por un número similar de pacientes intervenidos por una patología benigna distinta de la EPSC, excluyendo la obesidad mórbida.

Se define como retardo de cicatrización la falta de epitelización parcial o total de la herida operatoria luego de 30 días de la intervención. Se define como recidiva la descarga de material serohemático o purulento que se prolonga por más de 3 meses desde el procedimiento inicial o, bien, aparece en cualquier momento de la evolución de una herida ya cicatrizada. El seguimiento se concretó a través de controles en el policlínico de la especialidad y entrevistas telefónicas. Para la comparación de medias se utilizó la prueba $t$ de Student. Para estudiar una probable asociación entre variables categóricas se empleó la prueba del $\chi^{2}$ y el análisis de varianza (ANOVA) para comparar más de dos promedios. Los datos fueron procesados en el paquete computacional Stata 8.0 , considerando como significativo un $p \leq 0,05$.

En un período de 60 meses se intervinieron en forma electiva por una EPSC 74 pacientes, el 51\% hombres. El promedio de edad global fue 25,3 años (extremos 15-67; desviación estándar (DE): 11,85); el 10,8\% y el 5,4\% de ellos era mayor de 40 años y mayor de 50 años, respectivamente. El promedio de edad en las mujeres fue significativamente menor que en los hombres $(22,6$; DE: 10,6 vs 27,8 ; DE: 12,$5 ; \mathrm{p}=0,02$ ). El promedio del IMC fue 27,5 (DE: 5,03; extremos 19-47), siendo significativamente mayor en los varones $(28,6$; DE:5,3 vs 26,4 ; DE: $4,6 ; \mathrm{p}=0,03)$. Cuarenta pacientes fueron catalogados como hirsutos (54\%) sin diferencias en el promedio de edad ni del IMC entre ambos grupos. En cambio, hubo una clara asociación entre hirsutismo y el género, siendo los varones significativamente más hirsutos que las mujeres $(\mathrm{p}=0,014)$. Por otra parte, la distribución por edad según la categoría del IMC muestra que los pacientes con sobrepeso y obesidad tienen un promedio de edad superior que los pacientes con un IMC normal ( $p<0,0001)$. Al comparar el IMC de la serie con el grupo control podemos apreciar que el IMC promedio de ambos grupos es similar (IMC: 27,55; DE: 5,03 versus IMC: 27,19; DE: 4,00; $\mathrm{p}=0,31)$, con una distribución por edad y género similar (Tabla 1). Al categorizar los pacientes según el IMC (normales, sobrepeso y obesos) tampoco hubo diferencias entre el grupo de pacientes y el grupo control $(p=0,28)$. Como se aprecia en la Tabla 1, la distribución según el hirsutismo también es homogénea en ambos grupos.

La técnica de la resección asimétrica descrita por Karydakis fue empleada en 65 pacientes y la plastía de Limberg en 9. El tiempo operatorio promedio fue 32 minutos (extremos 20-45) para la primera técnica y 40 minutos (extremos 30-60) para la segunda. El tiempo promedio global de hospitalización fue 0,9

Tabla 1. Comparación de variables en casos y controles

\begin{tabular}{|lccc|}
\hline Variable & $\begin{array}{c}\text { Casos } \\
\mathbf{n}=\mathbf{7 4}\end{array}$ & $\begin{array}{c}\text { Controles } \\
\mathbf{n}=\mathbf{6 2}\end{array}$ & $\mathbf{p}$ \\
\hline $\begin{array}{l}\text { Edad promedio } \\
\text { (años) }\end{array}$ & 25,31 & 27,79 & 0,15 \\
$\begin{array}{l}\text { Género } \\
\text { (hombres/mujeres) }\end{array}$ & $38 / 36$ & $32 / 30$ & 0,97 \\
$\begin{array}{l}\text { IMC promedio } \\
\begin{array}{l}\text { Hirsutismo } \\
\text { (sí/no) }\end{array}\end{array}$ & 27,55 & 27,19 & 0,31 \\
\hline
\end{tabular}

$\mathrm{IMC}=$ índice de masa corporal. 
días (DE: 1,08; extremos 0-5) y en 29 pacientes $(39 \%)$ la intervención fue ambulatoria (todos con la técnica de Karydakis). La morbilidad global fue $11 \%$. La morbilidad fue mayor en los pacientes con IMC normal (5/22) que en los pacientes con sobrepeso y obesidad $(3 / 52)(\mathrm{p}=0,032)$. No hubo asociación entre el hirsutismo y la morbilidad en esta serie $(\mathrm{p}=0,54)$. El tiempo de seguimiento promedio fue 28,6 meses (extremos 6-60).

Conocer los factores involucrados en la etiopatogenia de la EPSC es determinante en el enfoque terapéutico. La resección radical de los tejidos afectados se ha justificado por la presencia de un "quiste infectado" con tejido granulatorio en una zona expuesta a la maceración y la infección ${ }^{1,17}$. De acuerdo con Bascom ${ }^{9}$, la EPSC es una condición patológica de la piel de la región sacrococcígea y por ello ha propugnado un tratamiento quirúrgico ambulatorio de menor envergadura con resultados variables en las series publicadas ${ }^{18-20}$. La obesidad se ha considerado como un factor predisponente, aunque la evidencia es controversial. Karydakis, destaca que el peso de los candidatos al servicio militar aumentó en 3,2 kilos en 10 años en Grecia, período en que la incidencia de EPSC se elevó de 4,9\% a 14,8\% ${ }^{4}$. Akinci, señala en soldados del ejército de Turquía que el porcentaje de pacientes con más de 90 kilos fue $39 \%$ en los 88 casos de EPSC versus $14 \%$ en 912 soldados del grupo control $(p<0,0001)^{13}$. Sin embargo, la frecuencia de sobrepeso y de obesidad en las series publicadas fluctúan entre $20 \%$ y $37 \% \%^{2,6,16,21}$, confirmando que esta condición se presenta en la mayoría de los casos en pacientes de peso normal. En nuestro estudio se aprecia que la obesidad no es un factor predisponente de la EPSC, lo que confirma los hallazgos de Cubukcu y cols 22 . Este autor compara 419 pacientes con EPSC con 213 controles, sin encontrar diferencias en el promedio del IMC de ambos grupos ni en las categorías según el IMC. A diferencia de nuestra serie, en dicho estudio la relación hombre/mujer fue 5:1 y no hubo diferencias en el promedio del IMC entre hombres y mujeres. En forma colateral, en nuestra serie no hemos encontrado que la obesidad y/o el hirsutismo aumenten los índices de morbilidad o de la recidiva de la $\mathrm{EPSC}^{23}$, lo que contrasta con lo publicado por otros autores ${ }^{24}$.

Aunque sin duda el hirsutismo es una condición asociada al género, ello no explica claramente la mayor incidencia de la EPSC en varones reportadas por autores de Grecia y Turquía, que fluctúa entre 3:1 y $14: 1^{10,13,22-24}$. Paradojalmente, el hirsutismo ha sido un factor etiopatogénico poco estudiado del "quiste pilonidal". Sin duda, el criterio para definir esta condición es subjetivo y, para disminuir la variación interpersonal, en nuestro trabajo el hirsutismo fue evaluado por uno de los autores. Akinci ${ }^{13}$, en un estudio de más de 1.000 pacientes, tampoco encuentra una asociación entre ambos, señalando que de hecho esta enfermedad también se presenta en personas rubias de pelo delgado y con escaso vello. En nuestra serie, el hirsutismo no aparece como un factor de riesgo para desarrollar la enfermedad, la que puede presentarse en personas lampiñas $\mathrm{y}$, aunque esta condición prevalece en los varones en las series extranjeras, en nuestra experiencia la EPSC se presenta con igual incidencia en ambos sexos.

Podemos concluir que la obesidad y el hirsutismo, como factor único o asociados, no son causas suficientes para explicar la aparición de la EPSC. Aunque los varones tienen un promedio de IMC superior y son más hirsutos que las mujeres, en nuestro medio la incidencia de la enfermedad es igual en ambos géneros. Otros factores como el estilo de vida han sido propuestos para explicar la alta incidencia de esta condición en varones jóvenes que están realizando el servicio militar ${ }^{13}$. El mecanismo exacto de cómo se desarrolla la EPSC es poco claro y esto afecta la elección de la técnica quirúrgica. Un estudio reciente realizado en Turquía en varones que realizan el servicio militar destaca el hirsutismo, la posición sentada por más de 6 horas al día y el hecho de bañarse 2 o menos veces por semana como factores de riesgo de $\mathrm{EPSC}^{25}$, aunque difícilmente se pueden extrapolar estas conclusiones al mundo civil.

\section{Tratamiento quirúrgico}

Existen más de 100 procedimientos quirúrgicos para el manejo de esta condición y con frecuencia se describen en la literatura nuevas variantes de algunas intervenciones que intentan alcanzar lo que se considera como una operación ideal, a saber, un procedimiento poco invasivo, de baja morbilidad, incapacidad laboral corta, baja recurrencia, estéticamente aceptable y ambulatoria. Existen 4 revisiones en la última década que coinciden en algunos aspectos relevantes: 1) En el tratamiento quirúrgico de la EPSC habitual, las técnicas cerradas tienen ventajas sobre las técnicas abiertas dado el prolongado período de cicatrización e incapacidad laboral de esta últimas; y 2) Dentro de las técnicas cerradas, las resecciones asimétricas (se incluye la técnica de Karydakis y variados tipos de plastías con rotación de colgajo) logran los mejores resultados en tiempo de cicatrización, reintegro laboral y tasas de recidiva ${ }^{17,21,26,27}$. Sin embargo, los estudios publicados no son comparables puesto que no evalúan los mismos parámetros en el análisis de resultados, aplican criterios disímiles en la medición del tiempo de cicatrización y, lo más importante, no existe 
una definición clara de lo que debe considerarse como recidiva, la que muchas veces se confunde e imbrica con la falla de cicatrización. A ello hay que agregar que los tiempos de seguimientos en muchas series son muy cortos y no existe un tiempo mínimo consensuado para medir los resultados alejados de la cirugía. La heterogeneidad de las series queda de manifiesto al comparar nuestra serie de 145 pacientes intervenidos por EPSC, de los cuales el $92,5 \%$ se intervino por presentar en algún momento de la evolución drenaje espontáneo o quirúrgico de un absceso con supuración intermitente, cifra que en la literatura extranjera promedia el $66 \%$ con un máximo de $75 \% \%^{9,12,16}$. La forma clínica de presentación en esta serie refleja probablemente lo tardío del tratamiento en nuestro medio y podría influir en los resultados del tratamiento quirúrgico, por ejemplo, si hay un predominio de pacientes intervenidos en la etapa de aumento de volumen sensible sin supuración, como lo muestran algunas series. Así, en la experiencia de Mentes $^{28}$, sólo 61 de 493 (12,3\%) pacientes intervenidos durante el servicio militar refería el antecedente de drenaje.

La técnica asimétrica de Karydakis, que pareciera cumplir con varios de los requisitos para ser considerada como la operación ideal, no es la más empleada en la actualidad según la revisión de la literatura especializada. Este autor griego propone corregir los factores predisponentes de la enfermedad mediante un procedimiento simple y reproducible, sin la necesidad de rotación de grandes colgajos o procedimientos de mayor envergadura. En un estudio sobre 6.545 pacientes intervenidos por esta causa señala que la causa de la recidiva es la persistencia de un surco inter-glúteo profundo y la localización medial de la cicatriz. Para corregir esta condición, propone una resección primaria asimétrica que aplana la fosita natal y deja la cicatriz en posición lateral ${ }^{10,11}$. De acuerdo a nuestra experiencia con la técnica ${ }^{16}$, destacamos algunos aspectos que pueden ser relevantes en los resultados: 1) Se traza una paramediana vertical a $2 \mathrm{~cm}$ de la línea media desplazada hacia el lado del orificio fistuloso o de la cicatriz y se talla una elipse que incluye toda la lesión; 2) El extremo caudal de la incisión es desplazada $1 \mathrm{~cm}$ más hacia lateral quedando a $3 \mathrm{~cm}$ de la línea media, de modo de alejar la herida del ano; 3) Luego de efectuada la resección con bisturí frío y efectuada la hemostasia se suelta el vendaje en $\mathrm{T}$ de los glúteos, se talla un colgajo graso de $2 \mathrm{~cm}$ de profundidad que se sutura en la línea media con puntos separados de Vicryl 3/0, lo que aplana la fosita natal; 4) Una segunda capa de puntos separados de Vicryl 3/0 entre el borde libre del colgajo medial y el borde lateral de la herida cierra el espacio muerto; 5) Hemostasia meticulosa. Habitualmente no requiere drenaje.
Cierre de la piel sin tensión con monofilamento 3/0 lo que permite apreciar una herida vertical ligeramente oblicua en posición lateral a la línea media; 6) Desde el traslado a su cama el paciente adopta la posición en decúbito supino, lo que, junto al vendaje compresivo, favorece la hemostasia en las primeras horas del postoperatorio; 7) La gran mayoría de los pacientes pueden ser intervenidos en forma ambulatoria ${ }^{29}$.

Las intervenciones con plastías (Limberg, Dufourmentel y otras) se reservan para la EPSC extensa con múltiples orificios fistulosos y extensión hacia el glúteo y para la EPSC recidivada. Hemos empleado la técnica de Limberg asimétrica 'modificada'30 que implica lateralizar la parte distal del colgajo que es oblicuo, evitando que cruce la línea media, lugar donde se producen los pocos casos de recidiva que se observan con esta técnica.

Con este protocololo de trabajo hemos intervenido en forma electiva 137 pacientes con EPSC en un período de 9 años, empleando la técnica de Karydakis en 127 pacientes y la plastía de Limberg en 10 casos. El promedio de edad de la serie fue 23,7 años (extremos 15-67), 70 mujeres y 67 hombres. En 20 casos existía una condición co-mórbida, destacando el tabaquismo en 7, neurológicas en 6 (tu cerebral, epilepsia, retardo mental), diabetes en 2 e hipertensión en 3 casos. El índice de masa corporal (IMC) promedio fue 25,9 (extremos 18,6-47) y el $56 \%$ tenía un IMC superior a 25 . La presencia de hirsutismo se registró como variable dicotómica en 73 pacientes (53\%). El 92,5\% de la serie tenía una historia de supuración crónica, drenaje de 1 o varios abscesos que fluctuaba entre 1 mes y 60 meses. E1 porcentaje de ambulatorización de la serie fue $60 \%$. El tiempo de seguimiento promedio fue 48 meses (extremos 6-96), durante el cual se comprueban 3 recidivas que fueron reintervenidas mediante una plastía de Limberg, con buena evolución posterior (se define como recidiva la descarga de material purulento o serohemático que se prolonga por más de 3 meses o que aparece en cualquier momento de la evolución de una herida ya cicatrizada). El estudio histopatológico de la pieza resecada mostró la presencia de inflamación aguda y crónica en todos los casos con antecedentes de abscedación, con tejido granulatorio, trayectos fistulosos en algunos casos, un manojo de pelos en el $45 \%$ y reacción gigantocelular a cuerpo extraño (RGC a CE) en el 38\%. No hubo correlación entre el tiempo de evolución de los síntomas, el número de abscesos drenados y la presencia de RGC a CE.

En conclusión, la técnica de Karydakis nos parece una técnica simple, reproducible para la EPSC habitual, la cual puede ser efectuada en forma ambulatoria con resultados satisfactorios a largo plazo. 
ENFERMEDAD PILONIDAL SACRO-COCCÍGEA: FACTORES DE RIESGO Y TRATAMIENTO QUIRÚRGICO

Tabla 2. Técnica de la resección asimétrica (Karydakis)

\begin{tabular}{|c|c|c|c|c|}
\hline Autor/año & $\mathbf{n}$ & Morbilidad herida op (\%) & Recidiva (\%) & Seguimiento promedio (meses) \\
\hline Kitchen $/ 82^{, 33}$ & 141 & 5,3 & 3,5 & 22 \\
\hline Anyanwu/98,34 & 28 & 10,7 & 3,5 & 22 \\
\hline Akinci/ $/ 00^{, 13}$ & 112 & 3,6 & 0,9 & 53 \\
\hline $\mathrm{Bessa} / 07^{35}$ & 82 & 7 & 0 & 20 \\
\hline Keshava/07,36 & 70 & 8 & 4 & 36 \\
\hline Bannura/10' & 127 & 9 & 2,4 & 48 \\
\hline
\end{tabular}

Tabla 3. Colgajo romboidal (Limberg y modificaciones)

\begin{tabular}{|c|c|c|c|c|}
\hline Autor/año & $\mathbf{n}$ & Morbilidad herida op (\%) & Recidiva (\%) & Seguimiento promedio (meses) \\
\hline Cubucku/00,24 & 114 & 2,7 & 5,3 & 24 \\
\hline Topgül/03,37 & 200 & 4,5 & 2,5 & 52 \\
\hline Mentes $/ 04^{\prime 38}$ & 238 & 0,8 & 1,3 & 29 \\
\hline Daphan/04 ${ }^{, 39}$ & 147 & 6,1 & 4,8 & 13 \\
\hline $\mathrm{Akca} / 05^{, 32}$ & 100 & 2 & 0 & 28 \\
\hline Akin $/ 08^{\prime 40}$ & 411 & 6,5 & 2,9 & 109 \\
\hline Polat $/ 11^{, 42}$ & 133 & 11 & 1,5 & 23 \\
\hline
\end{tabular}

Es fundamental lograr el aplanamiento de la fosita natal y dejar una cicatriz lo más alejada de la línea media. En una revisión de 17 estudios con 1.062 pacientes intervenidos con la técnica de Karydakis, Thompson y cols, señalan que las complicaciones de la herida operatoria (dehiscencia parcial, infección, fallas precoces) fluctúan entre $3 \%$ y $24 \%$, la incapacidad laboral va ente 1,5 y 4 semanas, el tiempo de cicatrización completa varía entre 1 y 64 semanas y la recidiva promedia el 3,9\% ${ }^{31}$. Estos resultados son similares a los obtenidos con las plastías de Limberg u otras en términos de infección, dehiscencia y recidiva. Por tratarse de procedimientos de mayor envergadura, las reservamos para aquellos pacientes con EPSC extensa, compleja o recidivados. La experiencia publicada con ambas técnicas se resume en las Tablas 2 y 3 . La infección de la herida operatoria y la dehiscencia superficial se han sumado bajo el concepto de morbilidad de la herida operatoria, dado las diferencias en los criterios utilizados para categorizar las complicaciones.

La mayoría de los trabajos publicados comparan la resección primaria (simétrica) con cierre en la línea media con la resección asimétrica de Karydakis y con la plastía de Limberg, demostrando la superioridad de ambas técnicas plásticas que sacan la cicatriz de la línea media $21,26,27,30-32$. Algunas mo- dificaciones del colgajo romboidal, están diseñadas para evitar que la cicatriz cruce la línea media. Faltan más y mejores estudios prospectivos al azar comparando la técnica asimétrica de Karydakis con la plastía de Limberg, que podrían confirmar o desafiar los hallazgos de Ersoy ${ }^{42}$. Este autor compara en forma aleatoria 100 pacientes, encontrando un mayor índice de infección de la herida operatoria con la técnica de Karydakis en comparación con el colgajo romboidal de Limberg $(26 \%$ vs $8 \%$ ), con cifras similares de hospitalización y tiempo de incapacidad laboral. Desgraciadamente no se señala el tiempo de seguimiento ni la recidiva en cada grupo.

\section{Referencias}

1. Da Silva JH. Pilonidal cyst: cause and treatment. Dis Colon Rectum 2000;43:1146-56.

2. Sondenaa K, Anderson E, Nesvik I, Soreide A. Patient characteristics and symptoms in chronic pilonidal sinus disease. Int J Colorectal Dis. 1995;10:39-42.

3. Rodríguez E, Contreras J. Quiste pilonidal: nuestra experiencia. Rev Chil Cir.1976;28:64-8.

4. Vergara JI, Azolas C, Contador J, Pérez-O G, Garrido $\mathrm{R}$, Jensen $\mathrm{C}$, y cols. Tratamiento quirúrgico del quiste pilonidal. Rev Chil Cir. 1991;43:44-6.

5. Blake P, Bardavid C, Guzmán H. Tratamiento quirúrgi- 
co del quiste pilonidal. Rev Chil Cir. 1997;49:692-7.

6. Jarufe N, Bannura G, Contreras J Saxton F, Marró P. Enfermedad pilonidal crónica sacrococcígea. Rev Chil Cir. 1999;51:66-71.

7. Kauer G, Correa R, Rojas H. Utilización del colgajo romboidal simple en el tratamiento quirúrgico de la enfermedad pilonidal sacrococcígea. Rev Chil Cir. 1999;51:623-6.

8. Hardaway RM. Pilonidal cyst. Neither pilonidal nor cyst. Arch Surg. 1958; 76:143-7.

9. Bascom J. Pilonidal sinus. Current Therapy in Colon and Rectal Surgery 1990; 32-9. Victor Fazio, MD, editor. First edition. BC Decker Inc.

10. Karydakis GE. New approach to the problem of pilonidal sinus. Lancet 1973;ii:1414-5.

11. Karydakis GE. Easy and successful treatment of pilonidal sinus after explanation of its causative processes. Aust NZ J Surg. 1992;62:385-9.

12. Destito C, Romagnoli A, Pucello D, Mercuri M, Marin AW. Pilonidal sinus: long term results of excision and closure technique. Review of literature G Chir. 1997; 18:441-6.

13. Akinci OF, Bozer M, Uzunkoy A, Duzgun SA, Coskun A. Incidence and aetiological factors in pilonidal sinus among Turkish soldiers. Eur J Surg. 1999;165:339-42.

14. Beck DE, Wexner SD. Fundamentals of anorectal surgery. London: WB Saunders 1998;225-36.

15. Buie LA. Jeep disease (pilonidal disease of mechanized warfare). South Med J. 1944;37:103-9.

16. Bannura G, Barrera A, Melo C, Contreras J, Soto D, Mansilla JA. Operación de Karydakis en el tratamiento de la enfermedad pilonidal sacrococcígea. Rev Chil Cir. 2005;57:340-4.

17. Allen-Mersh TG. Pilonidal sinus: finding the right track for treatment. Br J Surg. 1990;77:123-32.

18. Senapati A, Cripps NPJ, Thompson MR. Bascom's operation in the day-surgical management of symptomatic pilonidal sinus. Br J Surg. 2000;87:1067-70.

19. Mosquera DA, Quayle JB. Bascom's operation for pilonidal sinus. J R Soc Med. 1995;88:458-9.

20. Bacom J, Bascom T. Failed pilonidal surgery. Arch Surg. 2002;137:1146-50.

21. Chintapatla S, Safarani N, Kumar S, Haboubi N. Sacrococcygeal pilonidal sinus: historical review, pathological insight and surgical options. Tech Coloproctol. 2003;7:3-8.

22. Çubukçu A, Carkman S, Gonullu NN, Alponat A, Kayabasi B, Eyuboglu E. Lack of evidence that obesity is a a cause of pilonidal sinus disease. Eur J Surg. 2001; 167:297-8.

23. Sakr M, El-Hammadi H, Moussa M, Arafa S, Rasheed M. The effect of obesity on the results of Karydakis technique for the management of chronic pilonidal sinus. Int J Colorectal Dis. 2003;18:36-9.

24. Çubukçu A, Gonullu NN, Kaksoy M, Alponat A, Karu $\mathrm{M}$, Ozbay O. The role of obesity on the recurrence of pilonidal sinus disease in patients who were treated by escisión and Limberg flap transposition. Int J Colorectal Dis. 2000;15:173-5.

25. Harlak A, Mentes O, Kilic S, Duman K, Yilmaz F. Sacrococcygeal pilonidal disease: analysis of previously proposed risk factors. Clinics 2010;65:125-31.

26. Petersen S, Koch R, Stelzner S, Wendlandt T-P, Ludwig K. Primary closure techniques in chronic pilonidal sinus. Dis Colon Rectum 2002;45:1458-67.

27. Lee PJ, Raniga S, Biyani DK, Watson AJM, Faragher IG, Frizelle FA. Sacrococcygeal pilonidal disease. Colorectal Dis. 2010;10:639-52.

28. Mentes O, Bagci M, Bilgin T, Coskun I, Ozgul O, Ozdemir M. Management of pilonidal disease with oblique excision and primary closure: results of 493 patients. Dis Colon Rectum 2006;49:104-8.

29. Bannura G, Barrera A, Contreras J, Melo C, Soto C. Operación de Karydakis ambulatoria en el manejo de la enfermedad pilonidal sacrococcígea. Rev Chil Cir. 2009;61:256-60.

30. Cihan A, Ucan BH, Comert M, Cesur A, Cakmak GK, Tascilar O. Superiority of asymmetric modified Limberg flap for surgical treatment of pilonidal disease. Dis Colon Rectum 2005;49:244-9.

31. Thompson MR, Senapati A, Kitchen P. Simple daycase surgery for pilonidal sinus disease. Br J Surg. 2011;98:198-209.

32. Akca T, Colak T, Ustunsoy B, Kanik A, Aydin S. Randomized clinical trial comparing primary closure with the Limberg flap in the treatment of primary sacrococcygeal pilonidal disease. Br J Surg. 2005;92:1081-4.

33. Kitchen PR. Pilonidal sinus: experience with the Karydakis flap. Br J Surg. 1996;83:1452-5.

34. Anyanwu AC, Hossain S, Williams A, Montgomery AC. Karydakis operation for sacrococcygeal pilonidal sinus disease: experience in a district general hospital. Ann R Coll Surg Engl. 1998;80:197-9.

35. Bessa SS. Results of the lateral advancing flap operation (modified Karydakis procedure) for the management of pilonidal disease. Dis Colon Rectum 2007;50:1935-40.

36. Keshava A, Young CJ, Rickard MJFX, Sinclair G. Karydakis flap repair for sacro-coccygeal pilonidal sinus disease. How important is the technique? ANZ J Surg. 2007;77:181-3.

37. Topgül K, Ozdemir Z, Kilic K, Gökbayir H, Ferahköse Z. Long-term results of Limberg flap procedure for treatment of pilonidal sinus: a report of 200 cases. Dis Colon Rectum 2003;46:1545-8.

38. Mentes BB, Leventoglu S, Cihan A, Tatlicioglu E, Akin M, Oguz M. Modified Limberg transposition flap for sacrococcygeal pilonidal sinus. Surg Today 2004; 34:419-23.

39. Daphan C, Tekelioglu MH, Sayilgan C. Limberg flap repair for pilonidal sinus. Dis Colon Rectum 2004; 47:233-7.

40. Akin M, Gökbayir H, Kilic K, Topgül K, Ozdemir Z, 
Ferahköse Z. Rhomboid excision and Limberg flap for sacrococcygeal pilonidal sinus. Colorectal Dis. 2008;10:945-8.

41. Polat C, Gungor B, Karagul S, Buyukakincak S, Topgül $\mathrm{K}$, Erzurumlu K. Is oval flap reconstruction a good modification for treatment pilonidal sinuses? Am J Surg.
2011;201:192-6.

42. Ersoy E, Devay AO, Aktimur R, Doganay B, Ozdogan M, Gündogdu RH. Comparison of the short-term results after Limberg and Karydakis procedures for pilonidal disease: randomized prospective analysis of 100 patients. Colorectal Dis. 2009;11:705-10. 